\title{
A comparison of gaze behavior among elderly and younger adults during locomotor tasks
}

\section{FABIO E. FONTANA ${ }^{1}$ | ALEXANDRIA UDING ${ }^{2}$ | ANDREW CLENEDEN ${ }^{\mid}$LINDSEY CAIN | LEAN A. SHADDOX ${ }^{1}$ | MICK G. MACK ${ }^{1}$}

${ }^{1}$ School of Health, Physical Education, and Leisure Services, Psychomotor Behavior Laboratory, University of Northern lowa, USA | ${ }^{2 S}$ chool of Medicine, Washington University in Saint Louis, USA ${ }^{3}$ School of Pharmacy and Health professions, Creighton University, USA | ${ }^{4}$ Department of Nursing, COE College, USA.

Correspondence to: Fabio E. Fontana, Psychomotor Behavior Laboratory, School of Health, Physical Education, and Leisure Services, University of Northern lowa, 203 Wellness/Recreation Center, Cedar Falls, IA 50614, USA

e-mail: fabio.fontana@uni.edu

\begin{abstract}
AT A GLANCE
Older adults at high-risk of falling adopted visual behavior strategies that may increase their predisposition to falls: (a) older adults at high-risk of falling gazed at the ground ahead before stepping at a more immediate target. (b) older and younger adults made shorter fixations to targets during the fast-paced condition. Prioritization of future over current events and reducing the time available to extract information from the environment may increase the likelihood of falls among high-risk older adults.
\end{abstract}

\section{ABBREVIATIONS}

$\mathrm{ms}$ - milliseconds

PUBLICATION DATA

Received 8 April 2014

Accepted 10 May 2014

Published 10 July 2014
ABSTRACT Morbidity and mortality are possible outcomes of falls among the elderly. Understanding of potentially ineffective gaze behaviors adopted during aging may lead to strategies that can be useful in fall prevention programs geared towards older adults. Thus, the purpose of this study was to compare the gaze behavior of older adults to young adults during locomotor tasks requiring participants to change the direction and vary the speed of walking. Older adults were further divided into high- and low-risk of falling groups based on scores in the Berg balance scale and pre-established risk factor criteria. Gaze behavior was measured using the applied sciences eye tracking system as participants walked under four different conditions (straight-path/normal-pace, $45^{\circ}$ angle-path/normal-pace, $90^{\circ}$ angle-path/normalpace, and straight-path/fast-pace). A 3 (groups) X 2 (targets) X 4 (conditions) mixed design ANOVA was computed for each of the following dependent variables: target fixation duration, fixation to toe-off interval, and gaze-off to heel-contact interval. The results suggest that, independent of group or target, fixations on target were shorter, the faster participants walked. Results also revealed that older adults at high-risk of falling tended to move their gaze off the to-be-stepped-on target before actually making heel contact with the target, whereas the young and older adults at low-risk of falling did not. Based on these results we recommend warning older adults about the negative effects of walking speed on their ability to recognize and comprehend the challenges on the ground ahead by suggesting they slow down when walking. Another strategy is to train older adults to make heel contact with the ground before transferring their gaze to another aspect of the environment, which may serve to reduce the likelihood of tripping. Interventions to improve the gaze behavior of older adults at high-risk of falling should be tested.

\section{INTRODUCTION}

Falling is a common occurrence among older adults with approximately one-third of this population registering at least one fall per year. ${ }^{1,2}$ For older adults, the consequences of falling can be major including serious broken bones, paralysis, and death. ${ }^{2}$ Even when physical injuries do not occur, falls may result in an increased fear of falling. ${ }^{3}$ Fear of falling negatively impacts the quality of life by interfering with the willingness of older adults to participate in daily activities such as grocery shopping, travelling, dancing, and meeting friends.

The visual system is the only sensory system to consistently provide proactive information about the demands of the environment during locomotion. This information can be crucial for the prevention of falls among the elderly. Although several aspects of the visual system may affect our ability to prevent falls, studies focusing on gaze behavior seem particularly promising. ${ }^{4,5}$ Gaze refers to the location in the environment aligned with the fovea, corresponding to 
approximately 3 degrees of the entire visual field. The fovea is the area in the retina that allows us to see with great resolution and capture the detail of objects in the environment. Knowing how to properly direct gaze towards important elements in the environment may aid the successful execution of movements. In fact, gaze has been shown to be strongly associated with improved sport performance. ${ }^{7,8,9,10}$

Several studies have examined visual fields during locomotion. Patla and Vickers ${ }^{11}$ asked college students participating in the study to walk on level paths while stepping on 17 targets along the way. Results indicated that participants fixated on targets that were located two steps ahead of them. By keeping gaze approximately 2 steps ahead, participants were able to acquire information about the path before each step.

Similar findings where gaze was used in a feedforward manner to predict future stepping locations were also found. ${ }^{5}$ The authors ${ }^{5}$ investigated the gaze behavior of elderly and younger participants as they stepped onto and off a raised platform. The findings indicated that participants made saccades to the floor ahead of the platform before the step onto the platform was initiated. The authors suggested that saccades to the floor ahead of the platform were used in a feedforward manner to prepare for stepping off the platform. Although both groups made saccades to the floor ahead of the platform, the older participants initiated the saccades significantly earlier than their younger counterparts. This earlier initiation of saccades may give older adults who have experienced cognitive decline more time to prepare for platform descent. However, as suggested by the authors, early initiation of saccades also underscores the difficulty older adults may have when trying to recover after tripping when stepping on or off a platform.

Complementing previous findings, Zietz and Hollands 6 found feedforward planning in stair walking ${ }^{6}$. In the study, young and elderly adults were asked to ascend and descend a flight of stairs while gaze was tracked. Both groups used gaze in a feedforward manner by generally fixating 3 steps ahead as they walked up or down the steps. Interestingly, gaze fixation duration results indicated that older adults took longer to extract information from the environment in order to plan future actions in this stair walking task.

Using a straight level path, Chapman and Hollands ${ }^{4}$ asked young and older adults to step onto two targets placed on the floor between the beginning and end of a path. Older adults were further divided into high- and low-risk of falling groups based on risk factors for falling (i.e. occurrence of previous falls during past year, prescription of anti-anxiety medications, etc.). It was found that older adults fixated on targets sooner and for a longer period than young adults. As with the previous studies, this increased fixation was attributed to a decline in cognitive ability. However, the most noteworthy finding was that older adults, especially older adults determined to be at high-risk of falling, moved their gaze to the subsequent target well before making heel contact to the to-be-stepped-on target while younger adults watched their step to the target at least until their heel was planted on the ground. The strategy used by older adults at high-risk of falling may be counterproductive as the planning of a future action may interfere with the quality of the current action. In fact, biomechanical analysis of their gait showed that older adults at high-risk of falling had less accurate and more variable foot placement on the target.

Marigold ${ }^{12}$ also found participants used vision in a feedforward manner fixating mostly on the areas of the path two steps ahead of them. However, in this study where participants were asked to navigate through an irregular terrain consisting of rocky, slippery, and tilted surfaces, older and younger participants exhibited similar gaze patterns. Lack of differences in gaze behavior for this task suggests that when the terrain is difficult, both older and younger adults use the same feedforward gaze strategies.

In summary, vision appears to be used in a feedforward manner when walking and climbing stairs. Furthermore, with the exception of one study ${ }^{12}$, differences between older and younger adults have been found. The identification of differences in gaze behavior between young and older adults with high and low risk of falling may lead to the design of appropriate prevention strategies. Although the increased risk of falling during aging can be attributed to several degenerative processes such as declining muscle mass, reaction time, cognitive abilities, and deterioration in vision acuity, vestibular and somatosensory systems, ${ }^{13,14}$ improper use of gaze may also be a significant contributor. Additionally, among all these factors, gaze behavior is the only one that could be completely reversed after an intervention ${ }^{15}$. Thus, it is the purpose of this study to compare the gaze behavior of young (20's) and older ( $>65$ years of age) adults during locomotor tasks requiring participants to change direction and speed of walking.

To our knowledge, there have been no prior investigations comparing the gaze behavior of younger and older adults during locomotor tasks where participants were required to walk at varying speeds and change direction. In general, we hypothesized that older adults would use different gaze behavior strategies than younger adults. More specifically, we hypothesized that older adults determined to be at high-risk of falling would start fixations earlier, perform longer fixations, and transfer gaze to the subsequent target sooner than the other two groups in all conditions. ${ }^{4}$ We also hypothesized that the speed of walking would have a significant influence on fixation times and gaze transfer away from the to-be-steppedon target. The faster participants walk, the shorter the time available to extract information from the ground. Thus, we specifically hypothesized that fixation times on the target would decrease and gaze transfer away from the to-be-steppedon target would occur earlier for all participating groups when completing the fast-pace walking task. 


\section{METHODS}

\section{Participants}

Six younger college students $\left(M_{\text {age }}=20.3 ; S D=0.82\right)$ and 12 older adults participated in the study $\left(M_{\text {age }}=77.2 ; S D=8.70\right)$. Although sample size was small, it was comparable to several other publications with sample sizes ranging from 4 to 7 participants per group $4,5,11,16,17,18$ To participate in the study, older adults had to be 65 years of age or older. Older adults were classified into high- $(\mathrm{N}=6)$ and low-risk of falling $(\mathrm{N}=6)$. Classification was based on the results of a balance test and self-reporting of risk factors. Balance was measured using the Berg Balance Test. ${ }^{19}$ All participants with scores $\leq 45.5$ were classified as high-risk of falling. ${ }^{3}$ Based on criteria modified from previous studies ${ }^{4,20}$, additional risk factors for falling were also used to classify older adults. To be in the high-risk of falling group, participants had to self-report at least two out of the following six risk factors: occurrence of a fall within the last year, previous fall requiring medical attention, 3 or more prescription medications, cardiovascular medications, anti-anxiety medication or sedative, and low body mass index. All older participants lived independently in the community.

Visual acuity and cognitive function were assessed to protect participants and avoid research bias. Visual acuity was measured based on the Snellen eye chart. All participants had binocular visual acuity of 20/40 or better. Cognitive function of older participants was assessed using the Mini-Mental state questionnaire. ${ }^{21}$ All older adults scored 26 or higher on the Mini-Mental state questionnaire, which is indicative of intact cognitive function. None of the participants required any assistive devices for walking (i.e. canes, walkers). Older participants were offered a gait belt; three of them decided to wear the gait belt throughout data collection. All participants provided written informed consent prior to data collection. The experimental procedures were approved by the University of Northern lowa Institutional Review Board.

Task

The walking task was adapted from Chapman and Hollands ${ }^{4}$, and consisted of a 5.52-meter straight path where subjects had to step onto two targets located at pre-determined distances (Anterior-Posterior separation $1.84 \mathrm{~m}$, Medial-Lateral separation $=.47 \mathrm{~cm}$ ) between the starting and finishing position. For this project, there were three different paths each consisting of a starting position, 2 targets, and an ending position. The linear distance between the starting position and target 1 , target 1 and target 2, and target 2 and finishing position were the same across paths (1.84 m). Targets consisted of green circular rubber poly spot markers, 9 inches in diameter, commonly used in athletic practice. The center of the targets was marked with a pink dot $1 \mathrm{~cm}$ in diameter. Path 1 was similar to the one used by Chapman and Hollands ${ }^{4}$ with two targets positioned in a straight path between the starting and ending position. For the second path, target 2 and the end targets were positioned at a $45^{\circ}$ angle to the right of target 1 and for path 3 the second and end targets were positioned at a $90^{\circ}$ angle to the right of target 1.

Instrumentation

The ASL mobile eye tracking system was used to measure gaze behavior. The ASL mobile eye consists of a pair of optics with two cameras mounted on it: eye and scene cameras. The eye camera measures gaze behavior and the scene camera captures the scene in front of the participant. The eye and scene images are superimposed by the ASL eye tracking system and give real time information about where in the environment line-of-gaze is located. Before any data collection was conducted, the mobile eye system was calibrated. The mobile eye-tracking system operated tetherlessly during data collection.

A third camera (Canon ZR900) recorded stepping movements so the gaze behavior could be associated to the correspondent gait phase. The Vision-In-Action software was used to integrate the images of gaze behaviors and stepping movement and analyze coupled gaze and stepping behaviors frame-by-frame. ${ }^{7,8,11}$

\section{Procedures}

The collection of gaze behavior started by asking participants to position themselves on top of the starting targets and walk towards the finishing targets so they stepped onto each of the two targets along the path. Participants were instructed to accurately step onto the targets. They were specifically told that the "ball of the foot should step right on top of the pink dot located on the center of the target". Participants were asked to perform a total of 24 trials under four different conditions: a. straight-path/normal-pace; b. $45^{\circ}$ angle-path/normal-pace; c. $90^{\circ}$ angle-path/normal-pace; d. straight-path/fast-pace. Participants were asked to walk at a self-selected speed during the normal-pace trials, and to walk as fast as possible while remaining accurate and safe during the fast-pace trials.

After instructions, each participant performed a total of 8 practice trials, two under each condition. After practice trials were completed, 16 experimental trials were performed, 4 in each condition. Some participants still had questions about the procedures during the first four trials, so only the last 12 experimental trials were used for data analysis. During experimental trials, a board $(56 \mathrm{~cm} \times 71.5 \mathrm{~cm}$ ) was placed in front of the participant's face blocking their view of the subsequent path. This procedure was used so trials had a consistent starting point. The board contained a written 
description of the conditions to be performed next. Participants were told that they were free to start the trial once the board was removed. The order of trials was randomly assigned to subjects using the Latin Square procedure.

Data Analysis

A 3 (groups) X 2 (targets) X 4 (conditions) mixed design ANOVA was computed for each of the following dependent variables: a) target fixation time; b) interval between initial fixation to a target and toe-off to step onto that target; c) interval between the gaze transfer away towards subsequent target and heel contact onto the to-be-stepped-on target. Group (young, older low-risk, and older high-risk) was the between-subject factor, and targets (target 1 and target 2) and conditions (straight-path/normal-pace, 45 angle-path/normal-pace, 90 angle-path/normal-pace, straight-path/fast-pace) were the within-subject design factors. To examine potential differences between groups in the speed of walking, a second 3 (groups) $X 4$ (conditions) mixed-design ANOVA with repeated measures for conditions was computed for the dependent variable speed of walking. The manipulation of walking speed was checked by a 3 (groups) X 4 (conditions) ANOVA with repeated measures on conditions. Follow-up tests using the Bonferroni procedure for within factors and the Least Square procedure for between factors were computed as necessary. All statistical analyses were computed via the use of SPSS version 16 (SPSS Inc., Chicago, IL).

\section{RESULTS}

Walking Speed Manipulation Check - Significant main effects for groups $\left(F_{1,15}=12.45 ; p<.01 ; \eta_{p}{ }^{2}=.62 ; 1-\beta=.99\right)$ and conditions $\left(F_{3,45}=114.86 ; p<.01 ; \eta_{p}{ }^{2}=.88 ; 1-\beta=1.00\right)$ were found. For groups, post-hoc comparisons indicated that young adults $(M=5146 \mathrm{~ms} ; S D=650.48)$ walked faster than low- $(M=6524 \mathrm{~ms} ; S D=848.97)$ and high-risk older adults $(M=$ $7677 \mathrm{~ms} ; \mathrm{SD}=1548.24)$. Post-hoc comparisons also indicated that straight-path/fast pace $(M=5406 \mathrm{~ms} ; S D=1247.97)$, straight-path/normal-pace $(M=6496 \mathrm{~ms} ; S D=1282.77), 45^{\circ}$ angle-path/normal-pace $(M=6779 \mathrm{~ms} ; S D=1433.76)$, and $90^{\circ}$ angle/normal pace $(M=7114 \mathrm{~ms} ; S D=1525.67)$ were all significantly different from each other.

Target Fixation Duration - Significant differences for total fixation times were found among conditions $\left(F_{3,45}=8.12 ; p\right.$ $<.01 ; \eta_{p}{ }^{2}=.35 ; 1-\beta=.99$; Figure 1). Follow-up post-hoc comparisons indicated that independently of group or target, target fixation duration was significantly shorter under the straight-path/fast-pace condition $(M=1260 \mathrm{~ms} ; \mathrm{SD}=503.51)$ than for the straight-path/normal-pace $(M=1404 \mathrm{~ms} ; S D=506.69), 45^{\circ}$ angle/normal-pace $(M=1486 \mathrm{~ms} ; S D=502.15)$, and $90^{\circ}$ angle/normal-pace $(M=1474 \mathrm{~ms} ; S D=568.88)$ conditions. Significant differences were also found between targets $\left(F_{1,15}=7.91, p<.05 ; \eta_{p}{ }^{2}=.35 ; 1-\beta=.75 ;\right.$ Figure 2$)$. Longer fixations were performed for target $1(M=1565 m s ; S D=$ 598.80) than for target $2(M=1247 m s ; S D=376.89)$. None of the other results for target fixation duration were significant.

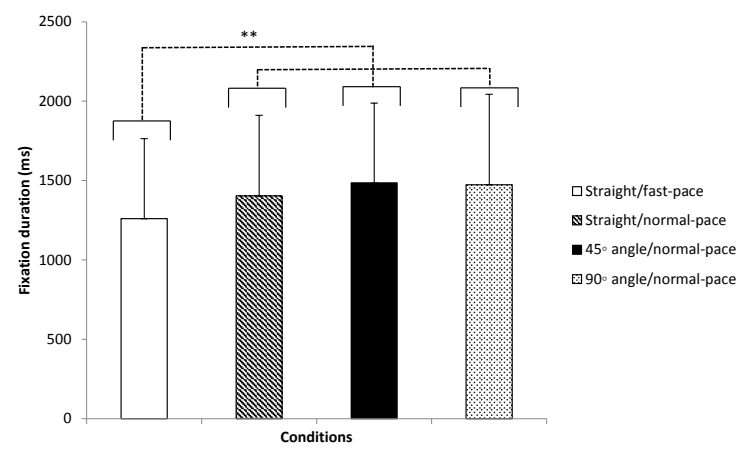

Figure 1. Mean (SD) target fixation duration by condition $\left({ }^{* \star} \mathrm{p}<.01\right)$

Fixation-on To Toe-off Interval - Results comparing the interval between initial fixation to a target and toe-off to step onto the target revealed a significant main effect for targets $\left(F_{1,15}=10,36 ; p<.01 ; \eta_{p}^{2}=.41 ; 1-\beta=.85\right)$. Longer fixations were performed for target $1(M=1141 \mathrm{~ms} ; S D=571.79)$ than for target $2(M=1247 \mathrm{~ms} ; S D=376.89)$. None of the other results for the fixation-on to toe-off interval were significant. 


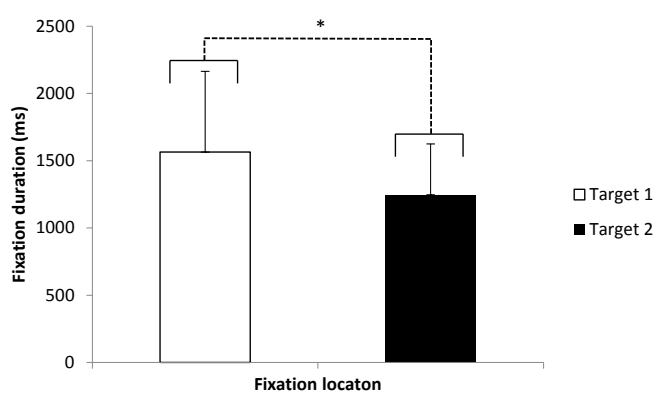

Figure 2. Mean (SD) target fixation duration by target $\left({ }^{*} \mathrm{p}\right.$ $<.05)$

Gaze-off To Heel-contact Interval - Significant differences were found between groups for the interval between the gaze transfer away from the to-be-stepped-on target and heel-contact onto that target $\left(F_{1,15}=4.63 ; p<.05 ; \eta_{p}{ }^{2}=.38 ; 1-\beta\right.$ $=.69$; Figure 3). Post-hoc comparisons indicated that, on average, the older adults at high-risk of falling transferred gaze away from the to-be-stepped-on target before stepping onto that target $(M=-106 \mathrm{~ms} ; \mathrm{SD}=256.57)$ while older adults at low-risk of falling $(M=104 \mathrm{~ms} ; S D=131.87)$ and young adults $(M=160 \mathrm{~ms} ; S D=107.66)$ transferred gaze only after stepping onto the target.

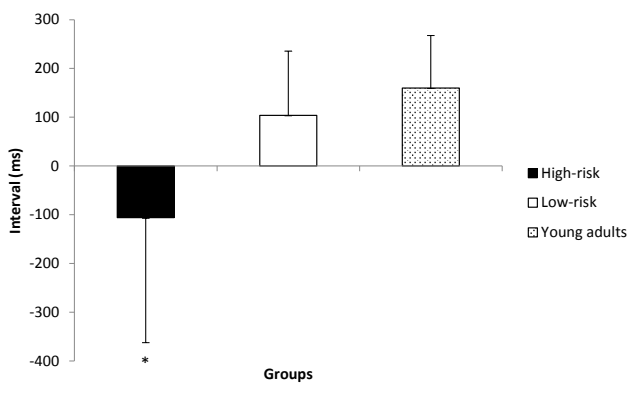

Figure 3. Mean (SD) gaze-off to heel-contact interval by group $\left({ }^{*} \mathrm{p}<.05\right)$

A main-effect for conditions was also significant $\left(F_{1,45}=3.42 ; p<.05 ; \eta_{p}{ }^{2}=.19 ; 1-\beta=.73\right.$; Figure 4). Post-hoc comparisons indicated that, independent of group or target, participants transferred gaze away from the to-be-stepped-on target more quickly for the fast-pace condition $(M=13 \mathrm{~ms} ; S D=223.46)$ than for the $45^{\circ}(M=89 \mathrm{~ms} ; S D=155.45)$ and $90^{\circ}$ angle conditions $(M=66 \mathrm{~ms} ; S D=211)$. None of the other results for gaze-off to heel-contact interval were significant.

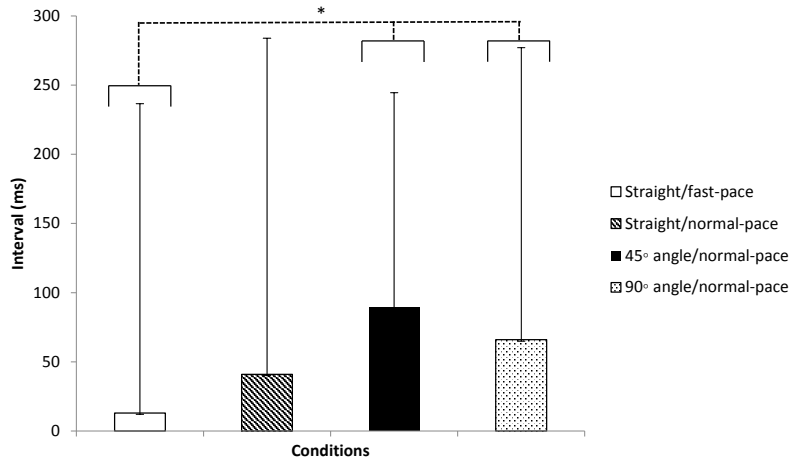

Figure 4. Mean (SD) gaze-off to heel-contact interval by condition ( $\left.{ }^{*} p<.05\right)$ 


\section{DISCUSSION}

The purpose of this study was to compare the gaze behavior of young and older adults during locomotor tasks requiring participants to change the direction of walking and to walk at different speeds. Because of potential differences in gaze behaviors due to differences in the aging process ${ }^{4}$, older adults were further separated into high- and low-risk of falling. It was our general hypothesis that gaze behaviors adopted by older adults would increase their predisposition of falling.

Findings from this study supported previous findings in which vision was used in a feedforward manner. $4,5,6,11$ Independent of groups, fixations to a target started well before participants made a step toward that target. While beneficial for all groups, this strategy is probably even more important for older adults who are at high-risk for falling since it gives them time to prepare in advance, thus reducing the likelihood of falling.

Speed of walking results indicated that walking times differed by condition, which confirmed the effectiveness of the speed manipulation. Results also revealed that younger adults walked significantly faster than low- and high-risk older adults. Furthermore, while not statistically significantly different, the times of the low- and high-risk older adults were in the expected order (i.e., $6524 \mathrm{~ms} \& 7677 \mathrm{~ms}$, respectively).

Contrary to expectations, there were no differences between groups concerning fixation times or fixation-on to toeoff interval. All three groups started fixations on the target at the same time and had the same target fixation duration. This combination of different walking times with no corresponding differences in fixation times or duration is noteworthy for a number of reasons. First, previous studies had indicated that high-risk older adults looked at targets sooner and fixated on targets for a longer period of time. ${ }^{4,16}$ Thus, the results of the present study contradict prior findings regarding gaze behaviors of high-risk older adults suggesting that additional research is warranted. Next, and perhaps more importantly, differences between groups' walk times without an equivalent difference in gaze behavior would suggest that the groups did actually differ in their gaze behavior. With shorter walk times and relatively similar fixation times, younger adults spent a higher percentage of their walk time fixating on the target as compared to the low-and high-risk older adults (e.g., 52.39\%, 40.77\%, and 40.09\%, respectively). It is interesting to note that younger adults, who are better equipped to prevent falls, spent a higher proportion of their walking extracting information from the ground.

Finally, older adults may have attempted to compensate for this reduction in time spent fixating by transferring their gaze before contact. Results indicated that older adults determined to be at high-risk of falling transferred their gaze away from the to-be-stepped-on target earlier than the other groups. On average, older adults at high-risk of falling transferred their gaze away from the to-be-stepped-on target before making heel contact with that target. Conversely, young adults and low-risk older adults transferred gaze away from the to-be-stepped-on target only after making heel contact with that target. That was true for both targets 1 and 2. Chapman and Hollands ${ }^{4}$ found a similar pattern of results and suggested that the prioritization of future events over current movements may increase the likelihood of falls among high-risk older adults.

As predicted, the time spent fixating on the targets was shorter for the fast-paced condition as compared to all of the normal-paced conditions (straight-path/normal-pace, $45^{\circ}$ angle, and $90^{\circ}$ angle). This was true for all three experimental groups. While perhaps not troublesome for younger adults, this decrease in length of gaze may be problematic for older adults, especially for high-risk older adults because it may increase their risk of falling due to the reduction in time available to extract information from the environment. Previous research has indicated that a decline in cognitive capacity $^{14}$ and poor balance are likely to explain why more time is required for older adults to judge the safety of the ground in a feedforward manner when walking.

While informative, this study was not without limitations. For example, it is possible that the instructions given in the current study placed more emphasis on stepping accuracy, which led to no differences being found between groups relative to when they started fixating on the target and for how long. While participants were instructed to make accurate steps to targets in previous investigations, the current study went further by clearly marking the center of the targets and providing demonstrations of what were considered accurate and inaccurate steps. It is possible that under our instructions even younger adults may have felt compelled to fixate on targets sooner and longer due to the emphasis on stepping accuracy. Although less likely, it is also possible that the locomotor tasks used in this investigation had levels of complexity comparable with the irregular terrain tasks used by Marigold ${ }^{12}$. Marigold ${ }^{12}$ had previously concluded that for tasks with high levels of complexity, both older and younger adults display the same gaze behaviors.

Another possible limitation concerns the finding that fixations to the first target occurred sooner and lasted longer than fixations to the second target. In previous studies using similar methodologies, no fixation duration differences between targets were found. ${ }^{4,16}$ The procedure used in this study, had researchers placing a board in front of the participant's face to block their view of the paths before they could start walking. This may have been the reason why several participants in the different groups started their fixation to the target even before making their first step out of the 
initial position. Since participants then walked continuously along the path, this additional fixation time was not evident for the second target.

\section{CONCLUSION}

In conclusion, results of the present study suggest that several gaze behaviors adopted by older adults, and more specifically high-risk older adults, may actually increase their predisposition to falls. Based on these findings, we offer a number of practical suggestions that may be used to help reduce or eliminate these unsafe walking behaviors. First, we strongly encourage older adults at high-risk of falling to walk at a comfortable self-selected pace even when rushed by external events. This strategy may be especially important in environments where it is more difficult to extract information such as cluttered environments or in snow covered ground. Slowing down or even stopping allows the brain more time to process visual information from the ground ahead, which is needed as one's cognitive abilities decline and balance diminishes. Another strategy that could be useful for preventing falls is to train older adults to make heel contact with the ground before transferring gaze to another aspect of the environment. By maintaining their gaze longer onto where they are currently stepping and fixating on important aspects of the ground longer, this may serve to reduce the likelihood of tripping as well as increase their ability to recover after tripping. Tripping has been previously identified as a problem for older adults. ${ }^{5}$

Future studies should focus on the trainability of gaze for locomotor tasks replicating more natural environments such as homes, grocery stores, and shopping malls. Finally, due to the high variance in gaze scores within the older groups found in the present study, additional investigations comparing the ability of variables such as depth perception and contrast sensitivity to predict early gaze transfer to traditional risk of falling categorization is recommended.

\section{REFERENCES}

1. Downton J. H., \& Andrews K. (1991). Prevalence, characteristics and factors associated with falls among the elderly living at home. Aging, 3, 219-28.

2. Tinetti, M. E. (2003). Preventing falls in elderly persons. The New England Journal of Medicine, 348, 42-49.

3. Ness, K. K., Gurney, J. G., \& Ice, G. H. (2003). Screening, education, and associated behavioral responses to reduce risk for falls among people over age 65 years attending a community health fair. Physical Therapy, 81, 631637.

4. Chapman, G. J., \& Hollands, M. A. (2006). Evidence for a link between changes to gaze behavior and risk of falling in older adults during adaptive locomotion. Gait \& Posture, 24, 288-294.

5. Di Fabio, R., Zampieri, C., \& Greany, J. F. (2003). Aging and saccade-stepping interactions in humans. Neuroscience Letters, 339, 179-182.

6. Zietz, D., \& Hollands, M. (2009). Gaze behavior of young and older adults during stair walking. Journal of Motor Behavior, 41(4), 357-365.

7. Harle, S. K., \& Vickers, J. N. (2001). Training quiet eye improves accuracy in the basketball free throw. The Sport Psychologist, 15, 289-305.

8. Panchuk, D., \& Vickers, J. N. (2006). Gaze behaviors of goaltenders under spatial-temporal constraints. Human Movement Science, 25, 733-752.

9. Vine, S. J., \& Wilson, M. R. (2010). Quiet eye training: effects on learning and performance under pressure. Journal of Applied Sport Psychology, 22, 361-376.

10. Wilson, M. R., Vine, S. J., \& Wood, G. (2009). The influence of anxiety on visual attentional control in basketball free throw shooting. Journal of Sport \& Exercise Psychology, 31, 152-168.

11. Patla, A. E., \& Vickers, J. N. (2003). How far ahead do we look when required to step on specific locations in the travel path during locomotion? Experimental Brain Research, 148, 133-138.

12. Marigold, D. S. (2006). Negotiating varying ground terrain during locomotion: insights into the role of vision and the effects of aging. (Doctoral dissertation, University of Waterloo). Retrieved from http://www.uwspace.uwaterloo.ca/bitstream/10012/2623/1/Marigold_PhD_Thesis_2006.pdf

13. Carter, N. D., Kannus, D., \& Khan, K. M. (2003). Exercise in the prevention of falls in older people: a systematic review of literature examining the rationale and the evidence. Sports Medicine, 31, 427-438.

14. Verhaeghen, P., Steitz, D. W., Sliwinski, M. J., \& Cerella, J. (2003). Aging and dual-task performance: A MetaAnalysis. Psychology and Aging, 18, 443-460.

15. Young, W. R., \& Hollands, M. A. (2010). Can telling older adults where to look reduce falls? Evidence for a causal link between inappropriate visual sampling and suboptimal stepping performance. Experimental Brain Research, 204, 103-113. 
16. Chapman, G. J., \& Hollands, M. A. (2007). Evidence that older adult fallers prioritize the planning of future stepping actions over the accurate execution of ongoing steps during complex locomotor tasks. Gait \& Posture, 26, 59-67

17. Di Fabio, R., Greany, J. F., \& Zampieri, C. (2003). Saccade-stepping interactions revise the motor plan for obstacle avoidance. Journal of Motor Behavior, 35(4), 383-397.

18. Hollands, M. A., \& Marple-Horvat, D. E. (2001). Coordination of eye and leg movements during visually guided stepping. Journal of Motor Behavior, 33(2), 205-216.

19. Berg, K., Wood-Dauphinee, S., Williams, J. I., \& Gayton, D. (1989). Measuring balance in the elderly: Preliminary development of an instrument. Physiotherapy Canada, 41, 304-311.

20. Di Fabio, R. P., Emasithi, A., Greany, J. F., \& Paul, S. (2001). Suppression of the vertical vestibulo-ocular reflex in older persons at risk of falling. Acta Otolaryngologica, 121, 707-714.

21. Folstein, M. F., Folstein, M. F., \& McHugh, P. R. (1975). Mini-Mental State: a practical method for grading the cognitive state of patients for the clinician. Journal of Psychiatric Research,12, 189-198.

Citation: Fontana FE, Uding A, Cleneden A, Cain L, Shaddox LA, Mack MG. (2014) A comparison of gaze behavior among elderly and younger adults during locomotor tasks. BJMB 8 (1).

Editor: Marcio A. Oliveira, University of Maryland, College Park, MD. USA

Copyright: (C) 2014 Foden et al and BJMB. This is an open-access article distributed under the terms of the Creative Commons Attribution-NonCommercial-NoDerivatives 4.0 International License which permits unrestricted use, distribution, and reproduction in any medium, provided the original author and source are credited.

Funding: This study was supported by a fellowship from the University of Northern lowa. EUA. The funders had no role in study design, data collection and analysis, decision to publish, or preparation of the manuscript.

Competing interests: The authors have declared that no competing interests exist. 